\title{
Hybrid
}

Revue des arts et médiations humaines

\section{Fictions fluides : les odyssées transmédiatiques de Daniel Canty}

\section{René Audet et Charles-Antoine Fugère}

\section{(2) OpenEdition \\ 1 Journals}

Édition électronique

URL : https://journals.openedition.org/hybrid/333

DOI : $10.4000 /$ hybrid.333

ISSN : 2276-3538

Traduction(s) :

Fluid fiction: Daniel Canty's transmedia odyssey - URL : https://journals.openedition.org/hybrid/418 [en]

Éditeur

Presses universitaires de Vincennes

Référence électronique

René Audet et Charles-Antoine Fugère, «Fictions fluides : les odyssées transmédiatiques de Daniel Canty », Hybrid [En ligne], 5 | 2018, mis en ligne le 18 décembre 2018, consulté le 15 avril 2022. URL: http://journals.openedition.org/hybrid/333 ; DOI : https://doi.org/10.4000/hybrid.333

Ce document a été généré automatiquement le 15 avril 2022.

Revue Hybrid 


\title{
Fictions fluides : les odyssées transmédiatiques de Daniel Canty
}

\author{
René Audet et Charles-Antoine Fugère
}

S'approprier le territoire, se laisser bercer par la géographie, aller à la rencontre de l'inattendu. Ces motivations profondément nord-américaines ont bercé la colonisation d'une terre en d'autres temps cartographiée par les Premières Nations; aujourd'hui toutefois, ces impulsions semblent définitivement surannées, voire surfaites. Il faut par conséquent une certaine candeur, un goût singulier du risque pour prétendre à une telle expérimentation du mouvement sur le territoire américain... tout comme il faut mobiliser une capacité inédite à en témoigner, autrement que par le récit de voyage un genre dont Michel Butor, il y a plus de cinquante ans, brisait les codes avec son Mobile $^{1}$, cette étonnante "étude pour une représentation des États-Unis ». S'engager dans une aventure similaire, à l'époque contemporaine, appelle une posture assumée autant qu'aérienne - c'est là l'a priori de Daniel Canty et de Patrick Beaulieu. Dans le cadre de ce qu'ils ont nommé des " odyssées transfrontières ", du Canada vers les ÉtatsUnis, ils se laissent guider par trois indicateurs différents : la migration des papillons monarques, l'orientation du vent et les indices de la chance. Ces projets participent d'un exercice, disent-ils, de science naïve $e^{2}$, où l'odyssée se matérialise par les traces, produites et recueillies, du parcours et de l'expérience vécue - mais tout autant par les déclinaisons multiples, décalées et polymorphes de son témoignage. C'est là l'aspect qui nous intéresse plus spécifiquement dans le cadre de cet article. Contrairement à la tendance actuelle du transmédia, qui par son renouvellement des formes de narration et de création issue des "mutations de notre société et de notre usage des nouveaux médias ${ }^{3}$ " fait aisément circuler les fictions sur des plateformes médiatiques complémentaires ${ }^{4}$, le monde littéraire reste davantage figé autour de l'idée que les fictions sont stables et qu'elles prennent une forme unique et pérenne - une fois le roman publié, il n'en circule ensuite que de simples variations de format (de la couverture rigide à l'édition de poche). Les littératures populaires exploitent certes ce filon médiatique, mais généralement elles opèrent plutôt dans la séquence (une série autour d'un personnage, par exemple) et dans le déploiement des univers. Les exemples des sagas populaires Harry Potter et Millennium illustrent cette propension actuelle à 
décliner la fiction sur différents types de supports médiatiques - romans, bandes dessinées, films, séries télé, jeux vidéo, fanfictions, applications pour appareils mobiles, etc. - selon une cohérence et une coordination savamment orchestrées ${ }^{5}$. De façon générale, de telles incarnations littéraires transmédiatiques mobilisées par la culture populaire ne semblant pas être associées au créneau de la littérature de champ restreint.

2 À cette tendance, l'auteur et artiste multidisciplinaire québécois Daniel Canty fait d'une certaine façon exception, proposant des projets littéraires qui se réinventent au gré des plateformes médiatiques qu'il convoque. Sans que ceux-ci rejoignent le fonctionnement coordonné et «impérialiste» des œuvres transmédiatiques ${ }^{6}$, Canty combine des fictions littéraires et des expositions artistiques, des projets de médiation culturelle et des vidéos, des sites web et des livres. Au cours des dernières années, Canty a notamment fait équipe avec l'artiste visuel Patrick Beaulieu pour élaborer et réaliser trois expériences de voyage singulières : Vector Monarca (2007), un périple où ils ont suivi les papillons monarques du Québec jusqu'au Mexique; Ventury (2010), une expédition où ils ont dérivé selon l'orientation du vent depuis Chicago jusqu'au sud-est des États-Unis ; puis Vegas (2013), un voyage où la chance a joué le rôle (arbitraire) d'un sélecteur de parcours. Les regards multipliés sur l'expérience, ses modalités d'échantillonnage, mais surtout l'appropriation diffractée de ces diverses traces nous paraissent exemplifier le caractère dominant de fictions qu'on dira fluides au sein de ces odyssées - car malgré un ancrage certain dans les artéfacts matériels du voyage, ce sont les mises en récit, fabulées autant que fabuleuses, qui s'imposent et se déclinent sur différents formats et supports. Le Web y joue non le rôle d'un point de chute, mais celui, déterminant, de relais, au sens d'un espace de passage et de transformation. De ce triple $\mathrm{V}^{7}$, nous insisterons sur le deuxième, Ventury, de façon à questionner les modalités d'une saisie médiatique du parcours, qui nous engagera à aborder les enjeux d'écriture et de représentation à travers la transmédiatisation des traces de cette odyssée.

\section{Mise en contexte}

3 Le projet Ventury a été initié par l'artiste visuel Patrick Beaulieu. Son objectif, original et ambitieux, était de suivre la direction du vent à partir de la ville de Chicago (the city of Wind). À bord d'une camionnette Ford Ranger bleue surnommée la Blue Rider et équipée d'une girouette et d'une manche à air, Beaulieu a convié quelques complices dans cette aventure qui les a conduits sur les routes américaines au gré de la direction du vent. L'expédition a duré vingt cinq jours, à l'automne 2010. Trois artistes se sont prêtés au jeu : l'architecte et paysagiste Alexis Pernet, l'artiste et écrivain Daniel Canty et l'écrivain Dauphin Vincent. Tour à tour, ils ont pris le rôle du copilote de Beaulieu, gardant à leur façon des traces de leur expérience dans un journal de bord et sur une carte routière. Ces immortalisations de leur épopée étaient pour eux «[le] reflet, [la] description ou [l']interprétation des vents qui ont soufflé, conjugués avec l'intensité de [leurs] affects ${ }^{8} »$. Le projet Ventury tenait du risque - Beaulieu prétend, après coup, qu'il aurait été prêt à abandonner la Blue Rider au large des océans si ç'avait été là la volonté du vent ${ }^{9}$. Outre le risque logistique d'une expédition soumise aux aléas de facteurs incontrôlables (le vol des papillons, le vent, la chance...), il y avait tout aussi bien celui de la rentabilité de l'odyssée, et surtout la rentabilité d'un projet artistique qui lui était 
immédiatement rattaché. L'objectif de l'expérience dépassait le simple circuit sur les routes américaines et la cueillette d'anecdotes à raconter. C'est que l'intégration d'une dimension résolument artistique à un tel périple engage la triple expérimentation associée à une perspective géopoétique, elle qui « [pose] sur le récit de voyage un triple regard, à la fois scientifique, philosophique et poétique ${ }^{10}$ ». L'écrivain adoptant une telle posture créatrice est particulièrement sensible à des éléments géographiques de son parcours (paysages, architecture, climat, etc.), intensifiant ainsi son rapport avec le monde. La géographie n'est toujours qu'une projection humaine sur l'espace, et comme Bertrand Westphal le signale à juste titre, toute géocritique requiert d'examiner les interactions entre les espaces humains et leur transcription littéraire - de débusquer les fondements culturels à cette saisie de l'espace par l'humain ${ }^{11}$. Plutôt que de retracer objectivement l'espace parcouru, celui-ci devient une source de réflexion et de création que le créateur peut capter à l'aide de médiums tels que l'appareil photo, le crayon, le pinceau. Pour le littéraire faisant l'expérience de la géopoétique, l'écriture ne correspond jamais à une transposition fidèle du voyage (ni ne vise à l'être), elle est au contraire toujours infléchie par une subjectivité propre à son auteur qui transpose son expérience et la prolonge par ses mots. Ainsi, la géopoétique appelle à la fluidité, répond au besoin de rendre compte du mouvement imprévisible du parcours plutôt que son aboutissement, d'une expérience singulière plutôt que factuelle.

Cette vision est tout à fait fidèle à l'esprit qui guide les créateurs de $V V V$, explicitement ouverts au travail de poétisation de l'expérience et du monde. Plus encore, c'est à une force d'abandon que les protagonistes se prêtent - une grande part de Ventury tenait de la fabulation, de l'imprévisible et du rêve : "Partir au vent. S'abandonner à des forces qui [les] dépassent. Obéir à une raison que la raison ignore ${ }^{12}$." L'accent n'était évidemment pas posé sur la destination, mais sur l'expérience du parcours, ses hasards, ses traces, ses effets sur les complices de l'odyssée. Afin de garder trace de leur périple, les artistes ont procédé à une forme d'échantillonnage: ils ont fait une sélection d'éléments isolés qui témoignent de tout ce qu'ils ont vu, entendu, vécu. Ils ont consigné des signaux nombreux de leurs péripéties, gardant une trace du nom de tel hôtel, de la conversation avec telle personne, du goût de tel repas. Pour procéder à cet échantillonnage, plusieurs modalités de captation du réel ont été utilisées, de même qu'ont été convoqués différents modes de mobilisation et d'exploitation de ce riche matériel.

\section{La saisie médiatique du parcours}

Dans les trois odyssées transfrontières, les façons retenues pour rendre compte des expériences ont émergé d'un esprit commun. Ayant sans doute pour fondement la capacité fabulatrice des artistes à faire des liens, à créer un sens artistique entre les éléments de leur voyage, cet esprit commun a d'abord pris la forme de contraintes. Ils se sont donné comme mandat de parcourir les routes à bord de voitures singulières, originales, conçues précisément pour leur projet : la Monarca Mobile (un camion postal transformé en véritable galerie d'art ambulante), le Blue Rider (un Ford Ranger muni d'une manche à air et d'une girouette) et la Magic Dart (une Dodge Dart ayant une roue de fortune sur le capot avant). À bord de ces véhicules emblématiques, les artistes ont emprunté un parcours particulier, dicté par un paramètre imprévisible : la migration des papillons monarques, la direction du vent et la chance. C'est donc au fil de 
l'errance, à travers des environnements fluctuants dont ils ont tenté de capter ici et là des éléments ponctuels, isolés, qu'ils ont essayé de se saisir, de manière discontinue, de la continuité même de l'expérience. Ce processus d'échantillonnage est complexe, démultiplié par la variabilité des supports médiatiques utilisés.

Modalité privilégiée combinant l'image, le son et le mouvement, la captation vidéo a fait partie des trois odyssées. Patrick Beaulieu filmait des scènes évoquant le thème de prédilection de chaque expédition. À titre d'exemple, dans le cas de Ventury, il a enregistré le mouvement ondulatoire d'un rideau sorti d'une fenêtre d'un appartement ou encore le frémissement des câbles électriques. La capture d'images était complétée par l'appareil photographique - que ce soit avec une caméra Polaroid (pour Vector Monarca) ou une caméra plus commune, les artistes prenant des clichés d'éléments du paysage auquel ils étaient confrontés. Les voyageurs ont également voulu garder une trace écrite de leur aventure. Chacun des périples était l'occasion pour Canty ou ses complices de rédiger un journal de bord ou un carnet de notes faisant état de leurs aventures. Ils ont donc écrit sur les conditions météo, les endroits où ils ont dormi et mangé, leurs rencontres, leurs positions géographiques. Parfois, ces carnets servaient à dessiner des schémas ou des croquis illustrant graphiquement quelques lieux rencontrés. La " table d'orientation ", mi-croquis mi-carte réalisée par Pernet et Canty, rassemble ainsi le nom de villes et lieux repères ("Mississippi [sic] ", "Cincinnati ", «Louisville »), en plus de comporter des annotations ponctuelles : « Où on est allé. / Ce qu'on a vu. / À qui on a parlé. / Ce qu'on a mangé. / Le temps qu'il faisait. / Les livres qu'on a trouvé $[s i c]$ »; "Quand le tracé d'une route commence à ressembler à celui d'une rivière ? Il faut avoir commencé par perdre la ligne d'un lac. ${ }^{13}$ "

7 Ces carnets offraient ainsi les conditions nécessaires pour tenir une réflexion à bâtons rompus sur l'expérience elle-même, permettant de transposer leur capture du réel en une fiction qui s'en inspire, distinction labile entre le témoignage et la fabulation. Par ailleurs, afin de garder une trace des fluctuations de leurs parcours, ils traçaient leur itinéraire sur une carte géographique, inscrivant le lieu de départ et le lieu d'arrivée de chaque journée de voyage. Les outils numériques complétaient leur arsenal médiatique: des traces ont été consignées à l'ordinateur, bien sûr, mais aussi le smartphone de Canty permettait, grâce à la géolocalisation de l'appareil, de transmettre à distance et en direct leur position géographique. Dans Vector Monarca, des lettres contenant des éléments épars de leur odyssée (bout d'une carte géographique, fragment d'un texte poétique, etc.) étaient ponctuellement envoyées à un webmestre resté à Montréal. Grâce à ces lettres, celui-ci érigeait à distance les bases du site web du projet.

8 Par ailleurs, les artistes se sont donné le mandat de recueillir différents objets en lien avec leur projet. Pour Vector Monarca, ils ont entre autres ramassé des ailes brisées de papillons monarques, mais aussi toute documentation papier ayant rapport avec ce thème (autocollants, napperons, macarons...). Pour le projet Ventury, ils ont rapporté avec eux des cartes postales qui évoquaient, de façon explicite ou pas, le thème du vent. Chaque jour, ils devaient se rendre chez un bouquiniste ou un antiquaire de la localité où ils se trouvaient. Cet exemple révèle aussi une variable supplémentaire, dédoublant l'imprévisibilité propre à leurs modes de saisie de l'expérience : l'aspect participatif. La méthode pour recueillir des cartes postales est exemplaire. Les commis des boutiques visitées jouaient le rôle d'interprètes, en ce qu'ils devaient décoder la demande de Beaulieu et ses acolytes et lui donner sens: "Auriez-vous des cartes postales du 
vent?» Le résultat était conséquent de la compréhension - littérale ou amusée - de cette demande saugrenue par les commis. La dimension collective est également rendue concrète par le système de relais entre accompagnateurs (Ventury) ou encore par la contribution d'un webmestre (Vector Monarca).

9 Dans le projet Vegas, cet aspect est autrement prépondérant. Tout au long du voyage, les artistes ont demandé à des individus qu'ils croisaient, au hasard de leur route, de faire tourner la roue de fortune installée sur la Dodge Dart (parmi eux, un policier plutôt médusé de l'installation sur la voiture...). En fonction du résultat obtenu, les artistes devaient ensuite emprunter une attitude particulière (l'innocence, la fortune, la contemplation...) et faire des liens avec ce qu'ils voyaient au courant de la journée. Dès lors, leur expédition était l'occasion de faire l'expérience de l'incident, les artistes se laissant guider par le hasard de leurs rencontres et les aléas de la nature (le vent ou la route migratoire des papillons). Associée à l'esthétique du flux par Gregory Chatonsky, la notion d'incident renvoie à un "sentiment que quelque chose nous déborde dans le champ du sensible et ne s'arrête jamais, coule indéfiniment comme si rien n'était plus autonome $\mathrm{e}^{14} »$. Cette esthétique tend précisément à provoquer des incidents, à se confronter à des événements incontrôlables, dictés par des forces étrangères, état d'esprit dans lequel sont placés Canty et ses acolytes. La dimension participative est donc déterminante dans la tentative de saisie du flux, qui constitue la trame de leurs expéditions. Les objets recueillis (ailes de papillons, autocollants...) correspondraient à un moyen d'extraire, par fragments, une parcelle de ce flux, et par le fait même cette cueillette constituerait une modalité pour en conserver la trace. C'est donc à partir de ces éléments épars arrachés à leur expérience - « la coupure fai[sant] partie intégrante du flux numérique ${ }^{15} »-$, fixés ici et là sur des supports multiples, que les artistes ont tenté de donner forme et corps à un voyage marqué par sa fondamentale imprévisibilité.

\section{Vers une transmédiation de l'expérience}

Donner forme, choisir l'angle, le cadrage, la focale. Ce sont les gestes du photographe, du sculpteur, du peintre, de l'écrivain. Nous mentionnions à l'instant les attitudes à emprunter par les voyageurs complices - on est à un jet de pierre des trois approches du réel expérimentées par Italo Calvino dans son recueil de fictions intitulé Palomar ${ }^{16}$, où les textes s'appuient tour à tour sur la description, le récit et la réflexion philosophique. On saisit bien là l'effet du dispositif, thème cher à cet ensemble d'articles, qui agit sur deux plans : l'action à réaliser sur le matériau; et l'effet de séquence, la temporalité du processus ${ }^{17}$. Modeler le réel, l'aborder selon une attitude, une perspective donnée (qu'elle soit choisie, contrainte ou imposée par le hasard), c'est là un geste au fondement même des odyssées de Beaulieu et de Canty, mais aussi un geste récurrent, réitéré, déclinant sous différentes espèces l'affabulation générée par l'expérience du voyage.

11 La démarche artistique associée aux odyssées transfrontières ne se situe pas dans un rapport de transformation simple du réel en une version esthétisée, qui viendrait dans un deuxième temps après avoir vécu les expéditions. Elle les encadre plutôt - des installations artistiques de Beaulieu, avec des ailes de papillons monarques, ont en effet précédé Vector Monarca, alors qu'un intérêt singulier pour le souffle (el soplo) préfigurait Ventury. La transformation matérielle mais tout autant symbolique des véhicules 
participe de cet engagement poétique dans l'aventure. Il est dès lors conséquent de voir le travail de constitution des traces - qu'on pense à la carte dite " géopoétique » de leur parcours, aux carnets griffonnés, aux nombreux échantillons d'ailes de papillon et à la captation vidéo de manifestations du vent - comme une emprise métaphorique sur le réel. C'est un geste certes immédiat alors que se déroule une odyssée à travers les ÉtatsUnis (l'événement se passe ici et maintenant, il faut retenir des traces de l'expérience en train de se vivre), mais c'est aussi un geste médié par la saisie esthétisante, qui place l'échantillon à distance du flux dont on l'extrait, un geste médié par le temps qui s'intercale - l'ancrage temporel d'origine s'efface au profit d'une multiplication des moments d'existence de ces traces. Le travail de constitution de ces traces n'est jamais objectif, n'est jamais neutre, mais la démarche de Beaulieu, de Canty et de leurs complices pousse un cran plus loin cette mise en forme, cette manipulation. Beaulieu multiplie en effet les installations artistiques à partir de son matériel vidéo et des objets amassés sur la route; avec Canty, il propose des projections, des expositions et des performances multimédia, dans différents lieux, que ce soit pendant les odyssées ou après, faisant alors vivre, par procuration et en différé, une version esthétisée d'une expérience incontrôlée de l'espace et de la culture américaine.

La distance et le détachement s'installent plus résolument par des formes décalées de ces odyssées. Proposés en phase ou en déphasage des expéditions, les trois sites web ${ }^{18}$ qui leur sont associés contribuent bien sûr à la pérennisation de ces événements à portée artistique - à preuve, le fait qu'on les consulte encore aujourd'hui, qu'ils jouent pour nous un rôle informatif. Leur fonction est toutefois inégale, le site Vector Monarca constituant pour son odyssée le complément le plus détaillé et le plus élaboré parmi les trois (alors que Ventury et Vegas, dans leur structure et leur ampleur, jouent plutôt le rôle de bandes-annonces d'un film qu'on ne peut qu'imaginer). Plus encore, au-delà de ces variations, c'est le déplacement médiatique lui-même qui s'impose, en ce qu'il bouleverse la transmission et l'expérience - celle du lecteur, du spectateur cette fois-là. Si l'approche informationnelle ou factuelle nous fait percevoir ces sites comme des stabilisations de matériel autrement voué à l'oubli, une lecture qui est attentive au jeu des supports atteste plutôt d'une dilution progressive du processus d'échantillonnage. En tant que traces de deuxième niveau volontairement constituées à partir des traces captées par les voyageurs, les sites web jouent pleinement de la remédiation (à la Bolter et Grusin $\left.^{19}\right)$, dans un rapport de distance et d'hommage, de rivalité et de réappropriation du support antérieur, du support d'origine.

Sur le site de cette première expédition ${ }^{20}$, la reproduction page par page d'un carnet de Vector Monarca nie la matérialité de l'objet tout en l'élisant au statut de trace, mais son avatar numérique peut être reçu dans sa dimension esthétique - la cartographie des pages, ses effets visuels et sa navigation image par image estompent le caractère informationnel de cette archive, au profit d'une perception distante de l'expérience vécue - distante par cinq degrés : la perception subjective, la rédaction en mots et images, l'inscription sur un support matériel, la numérisation en mode image du carnet et l'insertion des clichés dans l'architecture d'un site web. Ce conflit de proximité et de distance parait maximal lors de la consultation de ces sites web, consultation qui est modulée par des horizons d'attente propres à la culture numérique (qui se refusent d'emblée toute distance et décalage temporel), mais une consultation tout autant colorée par ce goût démesuré pour l'archive, que cette même culture numérique alimente à l'envi. 

esthétique des échantillons, des matériaux associés aux odyssées de Beaulieu et de Canty. L'ouvrage VVV est la somme des trois V, un " atlas géopoétique ${ }^{21}$ » selon leurs auteurs, qui fusionne, c'est-à-dire compare autant que distingue, les aventures initiées par Patrick Beaulieu et ses acolytes. À la fois prise de contact avec les expériences, à la fois regard méta sur la démarche artistique, le livre propulse encore plus loin la représentation par rapport au réel que celle-ci figure. Esthétisation des archives, mise en discours des attitudes, figuration des outils (les cartes sont au final inutilisables) : le rapport intermédiatique, du livre vers les supports qui ont accueilli ces contenus représentés, pourrait laisser plus d'un lecteur perplexe. Si ce n'est pas nécessairement le cas, si l'on fait sens de cette proposition, c'est que nous pouvons la saisir comme une réécriture, comme un déplacement. À travers toutes ses occurrences, l'odyssée dépasse le caractère itératif de ses formes d'expression pour entrer dans une boucle récursive mais avec un constant facteur de décalage - d'une fois à l'autre, une distance supplémentaire est imposée, laissant place à une part plus grande de subjectivité, de manipulation esthétique, de fiction. C'est en situant le projet global des VVV, ainsi que tous ses spinoffs, dans la dynamique médiatique qui lui est propre que l'on peut saisir cette logique, fluide parce que fondée sur la fluctuation, qui l'anime profondément.

Daniel Canty nous apparait sur ce point un créateur qui excelle au jeu de la variation transmédiatique. Les lecteurs bien informés savent que son curieux roman Wigrum ${ }^{22}$, une œuvre encyclopédique tout en feuilletés fictionnels, a pris naissance dans une matière fictive originellement utilisée comme remplissage d'un site web d'une jeune compagnie de création multimédia. Les fictions de Canty ont cette caractéristique d'être fluides, de migrer et se transmuer pour se cristalliser dans des manifestations distinctes, apparentées mais clairement autonomes. L'exemple de Ventury illustre fort bien cette fluidité. Canty a écrit un journal de bord au fil de sa participation à l'odyssée $\mathrm{du}$ vent dans l'est américain. L'ouvrage $V V V$, qui fait la synthèse des trois voyages, inclut une version du carnet qui occupe neuf pages du livre.

16 Pourtant, presque simultanément, Canty fait paraître, chez un éditeur littéraire, l'ouvrage Les États-Unis du vent, qui porte la mention générique « récit » (un indicateur habituel d'un discours autobiographique qui tolère néanmoins une certaine fictionnalisation). Ce récit de 283 pages rapporte à sa façon l'expérience de Canty au sein de l'odyssée Ventury, dont il en reprend tout aussi bien la chronologie sur une dizaine de jours que le principe du compte rendu de l'aventure vécue. Les neuf pages de carnet insérées dans VVV s'accordent à la facture de l'ouvrage (grand format, multicolonnes, images, travail graphique) et à son statut d'atlas géopoétique, à son statut de trace de traces (en engageant, tout comme l'ouvrage le fait dans son ensemble, la réflexion sur la démarche artistique); en comparaison, Les États-Unis du vent évacue la dimension graphique au profit d'un travail de la mémoire, mi-essayistique mifictionnel, qui met l'expérience à distance. La transformation opère peu à peu, depuis les carnets originaux (et inaccessibles) de Canty, jusqu'à un extrait sur le site web Ventury et les neuf pages à dominante descriptive dans VVV, puis l'expansion narrative et réflexive dans Les États-Unis du vent. Le substrat référentiel reste évidemment le même, ce dont témoignent certains passages qui persistent d'une version à l'autre ou qui sont dans leur rendu triturés par le jeu de l'écriture.

17 La portée, elle, s'appuie lourdement sur le support médiatique (qui définit pour chacun la circulation, l'audience et les codes de lecture) et sur la mutation même de la fiction - 
Les États-Unis du vent voyant émerger dans son déploiement, à titre de réécriture des états antérieurs, un large discours critique sur la culture et la société américaines. Les intertextes sont étonnamment variés, depuis les écrits d'Oliver Sacks et d'Oscar Wilde, en début de texte, jusqu'à " Un Canadien errant ", chanson écrite à la fin du XIX siècle par Antoine Gérin-Lajoie, le film de Michael Cimino, «The Deer Hunter », et de pseudoréférences télévisuelles comme "The Donut Monster of Northern Pennsylvania »... La version longue des carnets de route de Canty se constitue à partir d'un tissu vaste et dépareillé de mentions (souvent fugaces) à des contenus culturels variés - ceux du locuteur, bien sûr, mais aussi ceux qui émanent des espaces que les voyageurs s'approprient peu à peu. Un tel quilt culturel, conforme aux pratiques traditionnelles et populaires américaines, inscrit dans une exploration sociale et identitaire le parcours désœuvré de Beaulieu, Canty et consorts.

Étonnamment peu de place a été réservé dans cette étude à la route elle-même. Pourtant, comme le rappelle Victoria Stanton : «La route aux multiples possibles [...] devient [pour Beaulieu] un lieu interstitiel où se produisent des démonstrations performatives d'intuition, de nécessité et de sérendipité. Dans cette attitude performative s'épanouissent des considérations attentives envers un présent évanescent ${ }^{23}$." Se saisissant de la route, ce non-lieu pourtant infiniment chargé, incarnation éminemment concrète de ce flux numérique qui nous occupe dans le cadre de cet article, les odyssées transmédiatiques de Beaulieu et de Canty parviennent à charger de sens ce mouvement récursif qui les anime. Les occurrences de ces récits témoignant des traces de l'échantillonnage opéré sur le flux américain convergent toutes vers l'idée d'une saisie, impressionniste certes mais néanmoins convaincante, d'une société en déchéance - Canty décrit d'ailleurs ces aventures, au final, comme une sorte de "chronique des disparitions ${ }^{24}$ ». On peut aisément percevoir Les États-Unis $d u$ vent comme une sorte d'extrapolation de son journal de bord tenu durant son passage dans la Blue Rider (parce que l'enjeu de la fidélité à l'expérience vécue en est somme toute évacué). Ce rapport avec la fiction est conséquent de celui qu'entretient Canty avec le réel, voyant que le parcours même de ses odyssées suscitait la création d'une réalité parallèle ${ }^{25}$. L'artiste étant harnaché d'une palette de supports médiatiques multipliant les voix et les publics, ce n'est au final que l'inscription de la création dans un temps long qui permet une prise de distance, un déplacement, une réécriture et une réappropriation, nouvelle tout aussi bien que singulière, d'un matériel qui appartient en somme autant au réel qu'à l'imaginaire de ses protagonistes.

\section{Conclusion}

Par bien des aspects (médiatiques, processuels, génériques), le projet transmédiatique et transfrontière VVV auquel participe Daniel Canty s'écarte de la conception générale de l'œuvre littéraire en tant qu'unité définie, stable et réservée à un seul support exclusif. Canty travaille plutôt à partir d'une conception fluide de ses fictions, qu'il décline au gré de genres, de supports et de circonstances propres à en soutirer des variantes différemment expressives. À partir du cas de figure de Ventury, deuxième V de ce large projet, nous avons pu démontrer la propension de ses créateurs pour un échantillonnage du réel, lequel est mis à profit dans une écriture-réécriture des traces de ce périple. En elle-même, la démarche artistique choisie pour l'occasion est associée à une perspective fluctuante, subjective du voyage, qui est celle de la géopoétique - qui 
investit son constant aller-retour entre les référents géographiques et les manifestations spatialement ancrées des identités culturelles. La convocation d'une esthétique du flux s'est ensuite exprimée autant à travers les divers modes de saisie pris sur le vif de leur parcours (captations vidéo, photos, journal de bord, recueillement d'objets, etc.) qu'à travers les différentes incarnations médiatiques, à la fois autonomes et interconnectées, venant cristalliser a posteriori l'expérience du voyage (livres, sites web, vidéos et projets de médiation culturelle). Le dispositif entier, complexe par ses manifestations et les déplacements induits à la portée sémantique de celles-ci, paraît être tant un mécanisme de saisie du flux culturel et naturel américain que sa reproduction mimétique, récursive et tangente, sous la forme de fictions fluides.

Réside pourtant dans de tels projets une tension fondamentale, illustrant à quel point sont entrelacés les imaginaires de la technique, au moment de leur rencontre avec la culture numérique. Figure d'une part l'armada des mécanismes de la prévisibilité et de l'organisation avec l'image de la machine et du dispositif - lourds rouages imbriqués dont le fonctionnement obéit à une logique physique implacable. Ce sont les règles, les contraintes, les genres qui permettent la médiation d'un réel autrement informe. Ce sont les moyens que les explorateurs du triple $\mathrm{V}$ se sont donnés pour faire sens de leurs périples, moyens susceptibles de se saisir de cette Amérique sillonnée, cartographiée, décrite. Figure d'autre part la notion de flux, fortement teintée de son sens original associé aux liquides, à l'eau et à l'écoulement des rivières, que l'on transpose aux réalités pas encore catégorisées ou sériées, notion qui évoque la surcharge diluvienne et indomptée - de données des univers numériques. Le vent autant que la chance échappent à notre appréhension, et pourtant, conclut Canty à l'avant-dernier moment de ses États-Unis du vent, « [p]ourtant, le vent, comme le veut l'expression, a tourné en [leur] faveur. À force de lui faire confiance, [ils] commen[cent] peut-être à en contrôler le flux ${ }^{26}$ ?» C'est sur cette interrogation permanente, celle de notre capacité de contrôle - espérée ou supputée - sur le réel brut par nos pratiques artistiques, que reposerait une large part des esthétiques contemporaines.

\section{BIBLIOGRAPHIE}

BEAULIEU Patrick, Vector Monarca, 2007. [En ligne] http://vectormonarca.com/fr/index.php [consulté le 14 février 2018].

BEAULIEU Patrick, Ventury, 2010. [En ligne] http://venturyodyssey.patrickbeaulieu.ca/index.html [consulté le 14 février 2018].

BEAULIEU Patrick, Vegas, 2012. [En ligne] http://vegasodyssey.patrickbeaulieu.ca/index.html [consulté le 14 février 2018].

BEAUlieu Patrick et CANTY Daniel, VVV. Trois odyssées transfrontalières, Montréal, Éditions du passage, 2015.

BOLTER Jay David et GRUsIN Richard, Remediation. Understanding new media, Cambridge, MIT Press, 1999. 
BOUVET Rachel et MARCIL-BERGERON Myriam, « Pour une approche géopoétique du récit de voyage », Arborescences, ${ }^{\circ}$ 3, juillet 2013. [En ligne] https://www.erudit.org/fr/revues/ arbo/2013-n3-arbo0733/1017364ar/ [consulté le 15 janvier 2018].

BUTOR Michel, Mobile. Étude pour une représentation des États-Unis, Paris, Gallimard, 1962.

CALvino Italo, Palomar, Paris, Seuil, 1985.

CANTY Daniel, Wigrum, Chicoutimi, La Peuplade, 2011.

CANTY Daniel, « Patrick Beaulieu. Petite fabrique de l'âme », esse arts + opinion, $\mathrm{n}^{\circ}$ 75, printempsété 2012, p. 43. [En ligne] https://www.erudit.org/fr/revues/esse/2012-n75-esse088/66429ac/ [consulté le 14 février 2018].

CANTY Daniel, Les États-Unis du vent, Chicoutimi, La Peuplade, 2014.

CANTY Daniel et KLEIN Anne, « Entretien - Questions de temps. Création, archives et numérique », in Audet René (dir.), séminaire de la CÉFAN « Médiations numériques de la culture », Université Laval, ${ }^{\text {er }}$ décembre 2016, [En ligne] http://www.crilcq.org/fileadmin/CRILCQ/Video/ CEFAN_2016-12-01_Klein-Canty.mp4 [consulté le 14 février 2018].

Chatonsky Grégory, « Esthétique du flux », Rue Descartes, vol. 1, n 55, 2007, p. 86-99.

GROUPIERRE Karleen, « Enjeux des transmédias de fiction en termes de création et de réception », thèse de doctorat en arts, philosophie, esthétique, Université Paris 8, 2013.

GROUPIERRE Karleen, « Le transmédia : un dépassement du médium? », Appareil, vol. 18, 2017. [En ligne] http://appareil.revues.org/2403 [consulté le 15 janvier 2018].

JENKINS Henry, «Transmedia Storytelling 101 », Confessions of an Aca-fan, 21 mars 2007. [En ligne] http://henryjenkins.org/blog/2007/03/transmedia_storytelling_101.html [consulté le 13 avril 2018].

ORTEL Philippe (dir.), Discours, image, dispositif, Paris, L'Harmattan, 2008.

RYAN Marie-Laure, « Narration in Various Media », dans Peter Hühn et al. (dir.), The Living Handbook of Narratology, Hambourg, Hamburg University, octobre 2014. [En ligne] http:// www,lhn.uni-hamburg.de/article/narration-various-media [consulté le 13 avril 2018].

STANTON Victoria, « Trajectoires performatives. VVV : une trilogie d'odyssées transfrontières ", Inter, $\mathrm{n}^{\circ} 118$, automne 2014, p. 67-69.

WESTPHAL Bertrand, « Pour une approche géocritique des textes », Vox poetica, 2005. [En ligne] http://www.vox-poetica.org/sflgc/biblio/gcr.html [consulté le 16 avril 2018].

\section{NOTES}

1. Michel Butor, Mobile. Étude pour une représentation des États-Unis, Paris, Gallimard, 1962.

2. Daniel Canty, «Patrick Beaulieu. Petite fabrique de l'âme », esse arts + opinion, $n^{\circ} 75$, printempsété, 2012.p. 43. [En ligne] https://www.erudit.org/fr/revues/esse/2012-n75-esse088/66429ac/ [consulté le 14 février 2018].

3. Karleen Groupierre, «Le transmédia : un dépassement du médium ?», Appareil, vol. 18, 2017, p. 75. [En ligne] http://appareil.revues.org/2403 [consulté le 15 janvier 2018].

4. Communément attribuée à Henry Jenkins en raison de ses travaux séminaux sur la culture de la convergence et les pratiques transmédiatiques, la définition du transmedia (ou du transmedia storytelling) se cristallise autour de la formulation suivante, proposée par Marie-Laure Ryan : « 
The spreading of narrative content across multiple media platforms. » (« Narration in Various Media », in Peter Hühn [dir.], The Living Handbook of Narratology, Hambourg, Hamburg University, 2014. [En ligne] http://www.lhn.uni-hamburg.de/article/narration-various-media) [consulté le 15 janvier 2018]. De façon plus spécifique, dès 2007 sur son blogue, Jenkins proposait cette explication : «Transmedia storytelling represents a process where integral elements of a fiction get dispersed systematically across multiple delivery channels for the purpose of creating a unified and coordinated entertainment experience. Ideally, each medium makes its own unique contribution to the unfoldingof the story. " (« Transmedia Storytelling 101 », Confessions of an Aca-fan, 21 mars 2007. [En ligne] http://henryjenkins.org/blog/2007/03/ transmedia_storytelling_101.html [consulté le 15 janvier 2018].

5. Correspondant à ce que Karleen Groupierre désigne comme des «cross-média »: «Un ensemble de médias constitutif d'un même univers imaginaire global et cohérent qui sera diffusé de manière coordonnée et synchrone, et présenté comme un tout à un public choisi.» Voir Karleen Groupierre "Enjeux des transmédias de fiction en termes de création et de réception ", thèse de doctorat en arts, philosophie, esthétique, Université Paris 8, 2013, p. 31.

6. Et sans qu'ils ne se limitent par ailleurs à des médias numériques.

7. Un ouvrage-synthèse de ces trois projets a été publié : Patrick Beaulieu et Daniel Canty, VVV. Trois odyssées transfrontalières, Montréal, Éditions du passage, 2015.

8. Patrick Beaulieu et Daniel Canty, VVV. Trois odyssées transfrontalières, Montréal, Éditions du passage, 2015, p. 103.

9. Tel qu'évoqué lors d'une présentation de Canty et Beaulieu, intitulée « Jours de chance : trois odyssées transfrontières à travers l'Amérique », dans le cadre du colloque "La recherchecréation : territoire d'innovation méthodologique " tenu à l'Université du Québec à Montréal en 2014. [En ligne] http://www.methodologiesrecherchecreation.uqam.ca/?page_id=575 [consulté le 14 février 2018].

10. Rachel Bouvet et Myriam Marcil-Bergeron, «Pour une approche géopoétique du récit de voyage ", Arborescences, n 3, juillet 2013, p. 1. [En ligne] https://www.erudit.org/fr/revues/arbo/ 2013-n3-arbo0733/1017364ar/ [consulté le 15 janvier 2018].

11. " N'est-il pas temps, en somme, de songer à articuler la littérature autour de ses relations à l'espace, de promouvoir une géocritique, poétique dont l'objet serait non pas l'examen des représentations de l'espace en littérature, mais plutôt celui des interactions entre espaces humains et littérature, et l'un des enjeux majeurs une contribution à la détermination/ indétermination des identités culturelles ? (Bertrand Westphal, "Pour une approche géocritique des textes", Vox poetica, 2005. [En ligne] http://www.vox-poetica.org/sflgc/biblio/ gcr.html [consulté le 15 janvier 2018].

12. Daniel Canty, Les États-Unis du vent, Chicoutimi, La Peuplade, 2014, p. 11.

13. Patrick Beaulieu et Daniel Canty, VVV. Trois odyssées transfrontalières, Montréal, Éditions du passage, 2015, p. 114-115.

14. Grégory Chatonsky, «Esthétique du flux », Rue Descartes, vol. 1, n55, 2007, p. 86.

15. Grégory Chatonsky, « Esthétique du flux », Rue Descartes, vol. 1, n 55, 2007, p. 95.

16. Italo Calvino, Palomar, Paris, Seuil, 1985.

17. Chez Philippe Ortel, le dispositif est une «matrice d'interactions potentielles, une matrice interactionnelle ", renvoyant à sa nature de système complexe, à la fois technique, pragmatique et symbolique (Philippe Ortel [dir.], Discours, image, dispositif, Paris, L'Harmattan, 2008, p. 6).

18. Patrick Beaulieu, Vector Monarca, 2007. [En ligne] http://vectormonarca.com/fr/index.php ; Vegas, 2012. [En ligne] http://vegasodyssey.patrickbeaulieu.ca/index.html; Ventury, 2010. [En ligne] http://venturyodyssey.patrickbeaulieu.ca/index.html [consultés le 14 février 2018].

19. Jay David Bolter et Richard Grusin, Remediation. Understanding new media, Cambridge, MIT Press, 1999. 
20. La reproduction se trouve également dans l'ouvrage VVV, en pages $62 s q$, sous l'intitulé Carnet de science naïve. [En ligne] http://www.vectormonarca.com/fr/carnet.php [consulté le 14 février 2018].

21. Voir la fiche descriptive de l'ouvrage sur le site des Éditions du passage. [En ligne] http:// www.editionsdupassage.com/fr/livre/beau-livre/89/vvv [consulté le 14 février 2018].

22. Daniel Canty, Wigrum, Chicoutimi, La Peuplade, 2011.

23. Victoria Stanton, "Trajectoires performatives. VVV : une trilogie d'odyssées transfrontières», Inter, $\mathrm{n}^{\circ} 118$, automne 2014, p. 68.

24. Patrick Beaulieu et Daniel Canty, VVV. Trois odyssées transfrontalières, Montréal, Éditions du passage, 2015, p. 212.

25. Voir le propos que Canty tient sur la fiction et la réalité dans l'entretien qu'il accordait à Anne Klein (Daniel Canty et Anne Klein, «Entretien - Questions de temps. Création, archives et numérique », in René Audet (dir.), séminaire de la CÉFAN « Médiations numériques de la culture ", Université Laval, $1^{\text {er }}$ décembre 2016. [En ligne] http://www.crilcq.org/fileadmin/CRILCQ/ Video/CEFAN_2016-12-01_Klein-Canty.mp4 [consulté le 14 février 2018].)

26. Daniel Canty, Les États-Unis du vent, Chicoutimi, La Peuplade, 2014, p. 257-258.

\section{RÉSUMÉS}

S'intéressant aux processus de transmédiatisation de fictions littéraires, cet article étudie le cas des trois explorations artistiques et littéraires menées par Patrick Beaulieu et Daniel Canty, réunies dans l'ouvrage VVV. Procédant par échantillonnage du réel tout au cours de leurs périples sur les routes américaines, Beaulieu et Canty se trouvent à médiatiser leurs expériences par le truchement de diverses manifestations (expositions, sites web, œuvres littéraires). Les fictions de Canty affichent dans leurs occurrences multiples un caractère éminemment fluide, se déplaçant autant de lieu que de formes et de sens. L'examen comparé de ces variations illustre la prise de distance croissante des traces en regard de l'expérience dont elles sont issues.

\section{INDEX}

Mots-clés : culture numérique, dispositif, flux, littérature contemporaine, réécriture, transmédiatisation

\section{AUTEURS}

\section{RENÉ AUDET}

René Audet est professeur titulaire au département de littérature, théâtre et cinéma de l'Université Laval et membre du Centre de recherche interuniversitaire sur la littérature et la culture québécoises (CRILCQ). Ses travaux, subventionnés par le Conseil de recherches en sciences humaines du Canada, portent sur le roman et la nouvelle, la narrativité contemporaine, la littérature et la culture numériques, ainsi que l'édition en contexte numérique. Directeur de la revue savante numérique temps zéro et directeur de la collection « Contemporanéités » aux 
éditions Nota bene, il a codirigé plusieurs dossiers de revue et ouvrages collectifs sur la littérature contemporaine. Il dirige le laboratoire Ex situ. Études littéraires et technologie (http://ex-situ.info).

\section{CHARLES-ANTOINE FUGÈRE}

Charles-Antoine Fugère est étudiant à la maîtrise en études littéraires à l'Université Laval. Ses recherches s'orientent sur la littérature contemporaine et ses manifestations numériques. Il a entamé à l'automne 2017 la rédaction de son mémoire « Le lecteur contraint : prédétermination de la lecture en contexte numérique dans Déprise, The Stanley Parable et Chronique(s) d'Abîme ", financé par le Conseil de recherches en sciences humaines du Canada. Membre étudiant du CRILCQ (site Université Laval), il est notamment auxiliaire de recherche dans le cadre d'un projet sur le livre numérique. Il travaille aussi en tant qu'auxiliaire d'enseignement pour le cours en ligne « Approches de la culture numérique » donné à l'Université Laval. 